Originally published as:

Ratsch, B.A., Bock, C.-T.

Viral evolution in chronic hepatitis B: A branched way to HBeAg seroconversion and disease progression?

(2013) Gut, 62 (9), pp. 1242-1243.

DOI: 10.1136/gutjnl-2012-303681

This is an author manuscript.

The definitive version is available at: http://gut.bmj.com/ 


\section{Viral evolution in chronic hepatitis B: a branched way to HBeAg-seroconversion and disease progression?}

Boris A. Ratsch and C.-Thomas Bock*

Department of Infectious Diseases, Robert Koch Institute, Berlin, Germany

\section{*Correspondence to}

C.-Thomas Bock, PhD

Robert Koch Institute, Department of Infectious Diseases

Nordufer 20, 13353 Berlin, Germany

Phone: +49 30187542379

Fax: +49 30187542617

E-mail: bockc@rki.de

Key words: hepatitis B virus, viral evolution, viral diversity, HBeAg-seroconversion, immune response

Electronic word count: 1,138

Abbreviations: hepatitis C virus (HCV), human immunodeficiency virus (HIV), hepatitis B virus (HBV), chronic hepatitis B (CHB), hepatitis B e-antigen (HBeAg), general time reversible model (GTR), amino acid (aa), next generation sequencing (NGS) 
Evolution is a natural event that enables the animal and plant kingdoms to adapt and survive to changing environmental effects. The same evolutionary pressures also help to shape microorganisms including viruses. The evolutionary significance of virus infections has been a subject of discussion for decades; ${ }^{2}$ however, modern genomic analysis became an important focus of research when it became apparent that genetic variants of viruses could be linked to pathogenesis and disease progression. The first questions relating virus diversity to disease progression were provided by Pierre Lépine in 1938 who reported on the evolution of different strains of rabies viruses and a link between different characteristics of infectivity and virulence. ${ }^{3}$ Recent reports have also revealed the importance of viral evolution and genetic diversity in the pathogenesis of viral diseases in particular of RNA- (influenza virus, HCV) $)^{4,5}$ and retrovirus infections (HIV). ${ }^{6}$ Hepatitis B virus (HBV) utilizes a reverse transcription strategy that together with an error-prone viral polymerase has the potential to generate a large number of genetic variants. Chronic HBV (CHB)-infection follows four complex dynamic phases, namely immune tolerance, immune clearance, immune control/inactive state, and immune escape/reactivation that evolve over several decades. This scenario is usually accompanied by HBeAg-seroconversion. The frequency and severity of hepatitis flares can predict disease progression while early HBeAg-seroconversion confers a favorable outcome and late or absent HBeAg-seroconversion results in progression to severe liver disease, such as cirrhosis. The underlying mechanism leading to HBeAg-seroconversion is thought to be mainly due to immunologically mediated events. HBeAg-negative disease is often associated with the selection of precore/core mutations that develop because of immune pressure during immune clearance. ${ }^{7,8}$ However, studies of viral diversity and its association with viral evolution are infrequent and attempts to explain mechanisms leading to HBeAgseroconversion remain speculative.

In their paper published in GUT, Cheng and colleagues shed new light on the relationship between virus diversity, cumulative evolutionary changes and HBeAg-seroconversion in CHB 
(see page $\mathbf{X X}) .{ }^{1}$ They carefully explored the long term progression of HBV quasi-species relative to the time point of HBeAg-seroconversion. By performing a follow-up study (up to ten years) using preserved serum samples of CHB-patients, the viral diversity of HBeAgseroconverters was compared with non-seroconverters. In order to determine the initiation of viral evolution during CHB the authors estimated HBV-diversity by using pairwise distances under a general time reversible (GTR) model of nucleotide evolution. They observed a gradual increase of viral diversity in seroconverters that started seven years before HBeAgseroconversion occurred. Increases in viral diversity were accompanied by a generally higher evolutionary rate in seroconverters resulting in more distantly related virus species as illustrated in their phylogenetic analysis.

Cheng and colleagues then addressed the question of whether there is a preferred localization of sequence variations in the HBV-genome during viral evolution. Remarkably, genetic diversity was localized predominantly to the non-overlapping precore/core region whereas overlapping regions of the HBV-genome did not show a significant increase of diversity. Concomitant with rising viral diversity, the appearance of the precore stop codon mutation at nt1896 was significant more frequent in HBeAg-seroconverters before the development of HBeAg-seroconversion. These findings together with the decline of viral load and HBeAg-level years before HBeAg-seroconversion favors the hypothesis that increasing immune pressure leads to changes within the precore/core protein. Previous reports confirm this hypothesis showing that HBV-core promoter mutants (A1762T/G1764A and C1766T/T1768A) are predominant in late stage of HBeAg-seroconversion and can have major impact on HBV-replication and HBeAg-expression. ${ }^{7}$ Although the exact role of core promoter and precore stop mutations on HBeAg-seroconversion remains to be elucidated, at least some contribution is likely. 
The authors also reported on the observation of positive selection of amino acid (aa) substitutions at certain positions of the core antigen. In general, positive selection of aasubstitutions might occur as a result of a replication advantage or immunological pressure. ${ }^{9}$ Recent observations have shown a link between increasing viral diversity and the selection of several aa-changes in known T-cell epitopes during immunoclearance phase. ${ }^{8}$ Cheng et al. described five positions that are positively selected only in HBeAg-seroconverters (P5T, V13S, I27V, L60V, P135Q). While up to $100 \%$ of the sequences of individual patients are carrying at least one positive selected aa, approximately $40 \%$ had two or three positive selected sites. The observation of positive aa-selection is in line with a recently published study. ${ }^{10}$ Here, Cheng and colleagues demonstrated that respective aa-substitutions can occur years before HBeAg-seroconversion.

The appearance of these aa-substitutions during the immune clearance phase was also described in 1996; however, a correlation with HBeAg-seroconversion was not noted. ${ }^{11}$ Subsequently, cell culture experiments using recombinant HBV-genomes bearing single mutations at either position P5 or L60 revealed an extracellular phenotype with significantly reduced virion secretion but normal intracellular viral replication. ${ }^{12}$ Despite a potential disadvantage for the virus, like diminished secretion, it could be assumed that sufficient pressure may drive the virus to evolve towards the selection of aa-changes within specific sites. Since these arising mutations remain stably integrated after HBeAg-seroconversion the hypothesis of immune escape mutations seems to be well supported.

Cheng and colleagues have analysed 2,600 sequences in order to substantiate their hypothesis. Although cumulative viral evolutionary changes appear to precede HBeAgseroconversion, next generation sequencing (NGS) will bring additional and substantial indepth information. NGS may help to identify molecular processes leading to the onset of specific genetic changes in coding and core promoter regions during the natural course of CHB and during antiviral treatment. 
How does the study of Cheng and colleagues help us to understand the role of HBV diversity, the emergence of precore/core variants in the scope of cumulative viral evolution during HBeAg-seroconversion and thus affecting antiviral treatment success and outcome of liver disease? First, the authors demonstrated that the increase in HBV quasi-species is linked to mutations occurring predominantly in the HBV precore/core region. They hypothesized that specific immune pressure on T-cell epitopes may alter tolerance of CD4/CD8 T-cell reactivity whereas increased viral diversity can be responsible for stimulating the innate immune system. Of significance the selection of a greater number of sites in T-cell epitopes has recently been described supporting their role in host immunity. ${ }^{8}$ Secondly, mutations in the precore/core region can influence HBV capsid stability which in turn can affect virus maturation, virus secretion and viral load. Accordingly, complex immune- (alteration of immune response) and virus-mediated processes (replication fitness) can interact in harmony in shaping HBV evolution. ${ }^{8}$

The findings provided by Cheng et al. add new insights to our knowledge of the complex processes of viral evolution, viral diversity, and selection of HBV quasi-species in chronic hepatitis $\mathrm{B}$. We are still at the beginning of understanding these processes of viral evolution and the association with pathogenesis in general and in particular with CHB and HBeAgseroconversion. However, from the perspective of the HBV, viral evolution generating well adapted viral quasi-species is a perfect strategy to assimilate to changing environments including host barriers, host immune response, and antiviral therapy. 


\section{Competing interests None.}

Statement: "The Corresponding Author has the right to grant on behalf of all authors and does grant on behalf of all authors, an exclusive licence (or non exclusive for government employees) on a worldwide basis to the BMJ Publishing Group Ltd and its Licensees to permit this article (if accepted) to be published in Gut editions and any other BMJPGL products to exploit all subsidiary rights, as set out in our licence"

\section{Provenance and peer review}

Funding: This research received no specific funding.

Contributorship statement: Dr Ratsch and Dr. Bock equally contributed to the writing of the commentary.

\section{REFERENCES}

1 Cheng Y, Guindo S, Rodrigo A, et al. Cumulative viral evolutionary changes in chronic hepatitis B virus infection precedes HBeAg seroconversion. GUT 2012;XX:YY.

2 Anderson NG. Evolutionary significance of virus infection. Nature 1970;227:1346-7.

3 Lepine P. On the evolution of fixed strains of rabies virus. J Hyg (Lond) 1938;38:180-4.

4 Murcia PR, Wood JL, Holmes EC. Genome-scale evolution and phylodynamics of equine H3N8 influenza A virus. J Virol 2011;85:5312-22.

5 Farci P, Wollenberg K, Diaz G, et al. Profibrogenic chemokines and viral evolution predict rapid progression of hepatitis C to cirrhosis. Proc Natl Acad Sci U S A 2012;109:14562-7.

6 Herbeck JT, Rolland M, Liu Y, et al. Demographic processes affect HIV-1 evolution in primary infection before the onset of selective processes. J Virol 2011;85:7523-34.

7 Parekh S, Zoulim F, Ahn SH, et al. Genome replication, virion secretion, and e antigen expression of naturally occurring hepatitis B virus core promoter mutants. J Virol 2003;77:6601-12. 
8 Wang HY, Chien MH, Huang HP, et al. Distinct hepatitis B virus dynamics in the immunotolerant and early immunoclearance phases. J Virol 2010;84:3454-63.

9 Grenfell BT, Pybus OG, Gog JR, et al. Unifying the epidemiological and evolutionary dynamics of pathogens. Science 2004;303:327-32.

10 Wu S, Imazeki F, Kurbanov F, et al. Evolution of hepatitis B genotype C viral quasi-species during hepatitis B e antigen seroconversion. J Hepatol 2011;54:19-25.

11 Bozkaya H, Ayola B, Lok AS. High rate of mutations in the hepatitis B core gene during the immune clearance phase of chronic hepatitis B virus infection. Hepatology 1996;24:32-7.

12 Le Pogam S, Yuan TT, Sahu GK, et al. Low-level secretion of human hepatitis B virus virions caused by two independent, naturally occurring mutations (P5T and L60V) in the capsid protein. $J$ Virol 2000;74:9099-105. 